\title{
Healthy Young and Old Women Differ in Their Trunk Elevation and Hip Pivot Motions When Rising from Supine to Sitting
}

\author{
Neil B. Alexander, MD, Donna K. Fry-Welch, MS, PT, Lisa M. Marshall, BS, \\ Carolyn Chia-Fung Chung, BS, and Ann Marie Kowalski, BS*
}

OBJECTIVE: To describe the differences between healthy young and older women in regards to trunk elevation and hip pivot motions when rising from a supine to a seated position.

DESIGN: Cross-sectional comparison.

SETTING: University laboratory.

PARTICIPANTS: Two groups of healthy female volunteers: young adult female controls $(n=22$, mean age 23.5 years) and community-dwelling older female adults $(\mathrm{n}=17$, mean age 73.8 years).

MEASUREMENTS: Subjects were videotaped as they performed three controlled bed mobility tasks, starting from a supine position: (1) rising to a seated position at the edge of a firm plinth surface (SS); and rising to a seated position without moving to the edge of the bed while either (2) using hands (SUH) or (3) not using hands (SUNH). A series of movements involving the trunk were identified as subjects performed the SS task.

RESULTS: The older women were more likely to rotate and laterally flex their trunks, particularly in the later phases of the SS task. In addition, during the SS task, the older group was more likely to bear weight on their hip/gluteal area, particularly in the later phases, and more likely to use a broad pivot base, consisting of the hip and the elbow. While all young and old performed the SUH task, less than half of the older group could complete the SUNH task. Moreover, the subgroup of older adults who could not complete the SUNH task may have accounted for much of the differences between the young and the old on the SS task.

CONCLUSION: Healthy young and older women differ in their ability to rise from a supine to sitting position, primarily in the strategies used to elevate the trunk and facilitate a pivot. Trunk flexion ability likely contributes to the age group differences noted in rising. These data provide the basis for a biomechanical analysis of the critical body segment

From the "Division of Geriatric Medicine, Department of Internal Medicine, University of Michigan and the Geriatric Research Education and Clinical Center, Ann Arbor VA Medical Center; and the TPhysical Therapy Department, School of Health Professions and Studies, University of Michigan-Flint, Michigan. Supported by National Institutes on Aging Grants AG08808 and AG00519. Presented, in part, at the American Geriatrics Society Annual Meeting, Washing ton, DC, November 1992.

Address correspondence to Neil Alexander, MD, Division of Geriatric Medicine, 300 North Ingalls Building, Ann Arbor Ml 48109. motions and the strengths required to perform bed mobility tasks. J Am Geriatr Soc 43:338-343, 1995.

$\mathrm{D}_{6}$ ifficulty in transferring out of a chair or bed affects from 6 to $8 \%$ of noninstitutionalized persons aged 65 and over, or over 2 million older adult persons. ${ }^{1,2}$ For most functional assessment instruments, older adults are evaluated as independent or dependent in their transfers, and there is no analysis of the components of rising from a chair or bed or grading of rise performance. ${ }^{3}$ Quantitative evaluations of chair or bed rise motions can be applied in a biomechanical analysis to estimate the muscle strength and joint range of motion used to rise from a chair or bed. ${ }^{4}$

Common body motions used for various bed mobility tasks are described in classical physical therapy texts. ${ }^{5,6}$ These tasks are often described in a conceptual framework, such as the influence of righting reflexes on the rolling from a supine to side lying position. ${ }^{7}$ There are few empirical data supporting how these task descriptions are determined. ${ }^{5-8}$ One notable exception is a series of studies in which young subjects rise from a supine position on the floor to a standing position and roll from a supine position to a prone position. ${ }^{9-13}$ Whereas older adults (aged 50-69) perform bed mobility tasks more slowly than younger adults, ${ }^{14}$ few studies examine empirically which body motions are used by older adults to rise from a bed. Thus, our goal is to provide a more empirical and quantitative analysis of how older adults rise from a bed.

This analysis builds upon our previous work, ${ }^{15}$ where we found that general descriptions of body motions are useful in differentiating bed rise performance among older adults who have difficulty rising from a bed, older adults who do not have difficulty, and healthy young adults. The latter two groups differ in the use of their upper extremities during the rise. We speculate that healthy young and old adults use their upper extremities differently because of age-related differences in the ability to elevate the trunk and pivot at the hips while rising. Accordingly, the purpose of the present study is to describe more specifically how healthy young and old adults differ in the trunk and pivot-related motions they use while rising from a supine to a seated position. We hypothesize that older adults, compared with young adults, are less able to flex their trunks forward from a supine position. As a result, older adults are more likely to rotate and laterally flex their trunks 
and alter their pivot-related motions when rising from supine to seated position.

These data can be useful in determining the changes in bed mobility that occur as a result of aging. Specifically, age-related alterations in how older adults elevate their trunks might be considered in rehabilitation goals and therapeutic exercises for mobility-impaired older adults. These data also serve as the basis for biomechanical analyses of bed rise motions. These analyses support the development of new exercise approaches to compensate for age-related changes in bed rise motions and the development of alternative bed designs for mobility-impaired older adults.

\section{METHODS}

\section{Subjects}

Twenty-two young adult female controls (mean age $\mathbf{2 3 . 5}$ years, age range 20-27) and 17 community-dwelling older female adults (mean age 73.8 years, age range 64-80) volunteered for the study. Informed consent was obtained from all volunteers, and the protocol was approved by the hospital and university institutional review boards.

Young adult controls were recruited from a group of healthy undergraduate and physical therapy students. Community-dwelling older women were recruited from among those who had previously participated or had indicated a willingness to participate in university research. Both groups denied any history of significant neurological or musculoskeletal abnormalities and denied any difficulty in bed mobility or transferring.

All older adults underwent a standard medical history and physical examination, conducted by a physician geriatrician, which focused on the presence or absence of neurological and musculoskeletal abnormalities. ${ }^{16-18}$ Despite denying any significant neurological or musculoskeletal abnormalities, some older women had subtle findings on history and examination. Common findings in these older adults included the following: five subjects $(29 \%)$ had a history of a visual disease such as glaucoma, cataracts, or senile macular degeneration and yet distance vision in all of the older adults was $20 / 50$ or better in the best eye; six subjects $(35 \%)$ had experienced rare occasional pain or stiffness in the extremities, neck, or back in the past that was not present during testing; seven subjects $(41 \%)$ had asymmetric or decreased lower extremity reflexes; eight $(47 \%)$ had reduced vibration sense at the ankles. All but three older adults took daily prescription medications (mean of 1.5 prescription drugs per person), and the majority of these drugs were hormones (including estrogen and thyroid). Medications taken by the older adults that might have affected performance included antihypertensives, diuretics, and digoxin, but no subjects had postural symptoms or hypotension. Despite these findings, no older adults had focal decrements in extremity muscle strength and joint range of motion. They were also not obese as indicated by their body mass index (mean 24, range 22-28, in $\mathrm{kg} / \mathrm{m}^{2}$ ). All older adults indicated that they were righthanded and that they participated regularly in walking and exercise programs.

\section{Equipment}

Subjects started from a supine position on a flat plinth measuring approximately $2 \mathrm{~m}$ long and $1 \mathrm{~m}$ wide. The floor to plinth surface height was approximately $0.8 \mathrm{~m}$.
A video camera was placed on top of a tripod, approximately $2 \mathrm{~m}$ high, inferior and lateral to the left side of the plinth. This camera was located $2.8 \mathrm{~m}$ from the left inferolateral corner of the plinth and was angled downward at approximately 35 degrees, such that the subject was in full view for the supine position, the side-lying position, and seated position at the edge of the plinth. Using these settings, an overall view of all experimental trials could be accomplished without moving the camera. In addition, a mirror approximately $1.5 \mathrm{~m} \times 0.5 \mathrm{~m}$ was placed to the subject's right side, thereby enabling the subject's right upper and lower extremities to be visualized by the camera.

A videocassette recorder with slow motion and pause controls was used to facilitate the data acquisition described below.

\section{Protocol}

All subjects adhered to the following protocol. After being centered on the plinth, subjects started from a supine position with knees and hips extended, feet together, and arms at their sides. After a countdown, subjects were to rise at a comfortable rate to a seated position at the edge of the left side of the plinth (SS task). Subjects were then told to rise up to a seated position while keeping their legs in the plinth area and not moving their legs to the plinth edge. These "sit-ups" were performed under two circumstances. Subjects were first allowed to use their hands in any preferred manner (sit-up with hands or SUH) and then were told to keep their arms folded across their chest (sit-up with no hands or SUNH). Subjects were instructed not to grab the edge of the plinth surface during any part of the rise or "sit-up" tasks, but they were free to use any other motion necessary to complete the task. One practice trial was performed for each of the SS, SUH, and SUNH tasks, followed by three additional trials.

\section{Supine-to-Sit(SS) Task Descriptors}

Based on data from previous studies, ${ }^{9-13,15}$ a set of trunk and pivot-related motions used to rise from supine to sitting (SS task descriptors) were identified.

Motions were thought to differ during different phases of the SS task. Accordingly, trunk flexion was identified in relation to the phases of completion of a pivot of the hips and pelvis. The pivot was defined to begin with the onset of pelvis motion (see Figure 1a), as indicated by abduction of the ipsilateral thigh, and was subsequently described by estimating the degrees of thigh abduction from the initial start position. There were three phases of the pivot: early (the first 30 degrees of thigh abduction from the initial position), middle ( 30 to 60 degrees of thigh abduction), and late pivot (greater than 60 degrees of thigh abduction). The pivot was completed when the ipsilateral and contralateral thigh reached 90 degrees and the legs dangled off the side of the plinth.

The pivot-related motions were identified in relation to the extent of trunk elevation off the plinth surface. The extent of trunk elevation was determined by estimating the angle made between the subject's trunk and the horizontal surface of the plinth in the sagittal/vertical plane (see Figure 1b). There were three phases of trunk elevation: early (the first 30 degrees of trunk elevation), middle (30 to 60 degrees of trunk elevation), and late (greater than 60 degrees of trunk elevation). 

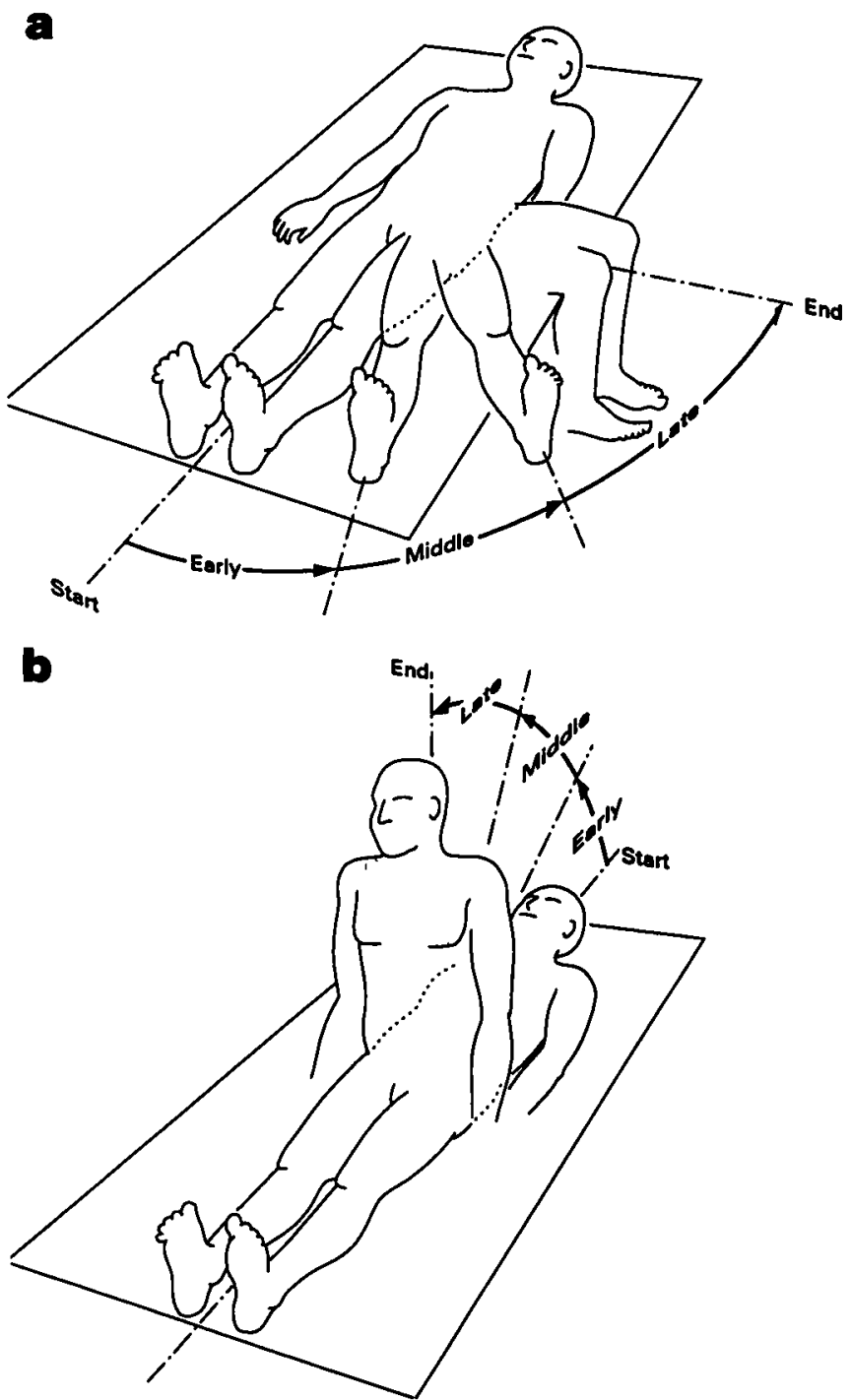

Figure 1. SS task descriptors are identified according to phases of completion of a pivot of the hips and pelvis (1a) and according to phases of completion of trunk elevation (1b).

1a. Phases of the pivot are determined by the extent of ipsilateral thigh abduction: early (the first 30 degrees of thigh abduction from the initial position), middle ( 30 to 60 degrees of thigh abduction), and late pivot (greater than 60 degrees of thigh abduction). The pivot is completed when the ipsilateral and contralateral thigh reach 90 degrees and the legs dangle off the side of the plinth.

1b. Phases of trunk elevation are determined by estimating the angle made between the subject's trunk and the horizontal surface of the plinth in the sagittal/vertical plane. There are three phases of trunk elevation: early (the first 30 degrees of trunk elevation), middle ( 30 to 60 degrees of trunk elevation), and late (greater than 60 degrees of trunk elevation).

The SS task descriptor items were defined as follows. Trunk flexion in the sagittal plane was noted when the head and trunk were aligned with and flexing towards the pelvis and lower extremities; trunk motion outside of the sagittal plane was noted as rotation or lateral flexion. Pivot variants included the use of the left hip and gluteal region for weight bearing and using the elbow in addition to the hip to make a broader base for the pivot.
Descriptor ratings within each category were generally independent, i.e., identifying one descriptor did not immediately determine the presence of another descriptor, with a few exceptions. During a particular phase of pivot completion, trunk flexion can begin primarily sagittally and later involve lateral flexion and/or rotation. Any descriptor could be noted during early, middle, or late phases of either the pivot or trunk elevation.

Further information regarding the development and use of these descriptors is available from the authors.

\section{Data Acquisition}

Two experimenters separately viewed videotapes of subjects performing the SS task and determined (rated) whether a particular SS task descriptor was present, specifically in relation to the extent of pivot completion and trunk elevation. In the few instances where there was disagreement in the presence of a descriptor (see section below on reliability), such as regarding the particular pivot phase of a descriptor, the videotape was independently reviewed by a third experimenter blinded to the scores of the other two experimenters. The third experimenter was used to break any "tie" scores, i.e., to determine a final consensus score. Use of a consensus score is useful in situations such as this where a gold standard is lacking, and where reliance on one rater might yield biased information. ${ }^{19}$ In addition, previous experience ${ }^{15}$ has shown that two independent ratings of bed mobility while using a descriptor-based rating system requires a third rater to determine a final score in situations where there is rater disagreement. This consensus score thus represents a composite rating based on more than one rater and more than one rating episode.

Although three trials of each task were performed, only the second trial was used for analysis. In previous studies, some intertrial variability was present for descriptors characterizing bed rise difficulty, perhaps reflecting a practice effect. ${ }^{15}$ For this reason, the second trial, instead of a mean of all trials, was used for the analyses.

In addition to identifying descriptors, the SS task was timed from motion onset to apparent task completion. SS task time was determined by an experimenter using a stopwatch and with the use of an inset timer on the video record. Start time was designated as the onset of any motion contributing to the task in the head, shoulder girdle, pelvis, or extremities. Motion was considered complete when trunk sway ceased while the subject was seated at the edge of the plinth.

Motions of body segments heralded the onset of the SS task performance. Initiation of the SS task was noted either by neck flexion, upper extremity motion, lower extremity motion, or any combination of the these motions.

\section{Data Analysis}

Group mean SS task completion time was compared between the young and old using independent $t$ tests. The percent of consensus SS descriptors (for each trunk elevation and pivot phase), the percent use of a body segment to initiate the SS task, and the percent able to complete the SUH and SUNH tasks in the young and old groups were compared using Fisher's exact test. The Fisher's exact test was also used to compare the percent of descriptors in those older women able to complete the SUNH task $(n=6)$ with those unable to complete the SUNH task $(n=8)$. 
Using the SS task ratings by the initial two experimenters, Cohen's Kappa scores for interrater agreement (between raters), intrarater agreement (same rater and same SS task trial on two separate occasions), and intertrial agreement (same rater, but two separate SS task trials) were assessed. Data were processed for the items noted below in Table 1, with a focus on the items that best differentiated the young and older women groups. Interrater reliability was analyzed in young $(n=20)$ and old $(n=14)$ subjects who did not roll onto their ipsilateral sides (see below). Intrarater and intertrial reliability were analyzed in smaller subsamples (intrarater young $n=10$, old $n=10$; intertrial young $n=17$, old $\mathrm{n}=12$ ).

All statistical comparisons were made using SYSTAT, a standard statistical software package. ${ }^{20}$

\section{RESULTS}

\section{Subjects Rolling onto Ipsilateral Side}

The SS task descriptors were identified for each subject, with the exception of a few young ( $2 / 22$ or $9 \%$ of group) and old $(3 / 17$ or $18 \%$ of group) subjects who clearly rolled onto their ipsilateral sides before elevating the trunk. These subjects followed the instructions as given, did not violate the protocol, and rose to a sitting position at the edge of the plinth. However, their motions differed markedly from the others in that these subjects did not flex their trunk in the sagittal plane and did not perform the hip pivot with the hip abduction and adduction as described above. These subjects used a completely different rise strategy, and, thus, the descriptor system was determined a priori to be too difficult to apply to these subjects. They were excluded from the SS task descriptor analyses only.

\section{Supine-to-Sit Task Descriptors}

Certain SS task descriptor items were commonly found in both young and old groups (see Table 1). Sagittal plane trunk flexion was common in both groups, especially at the beginning of the rise. During the pivot, weight bearing on the left gluteal/hip area was common, especially in the later phases of trunk elevation.

Significant differences between the young and old groups were as follows. The older women were more likely to use lateral trunk flexion and rotation, particularly in the late phase of the pivot. The older adults bore weight on their left hip/gluteal area more often, and at an earlier point of their trunk elevation, than the young. In fact, $75 \%$ of the young and $14 \%$ of the old bore weight on the left hip only in the late phase, compared with a combination of early, middle, or late $(P<.005)$. The older adults were also more likely to broaden their support base by contacting their elbow to the plinth. This was evidenced by the use of their elbow to broaden their pivot base during middle trunk elevation.

\section{Mean Task Completion Time}

Mean time to complete the SS task differed significantly between the young and old, whether roll subjects are included (young: mean $2.3 \mathrm{sec}, \mathrm{SD} 0.5$; old: mean $3.3 \mathrm{sec}, \mathrm{SD} 1.8 ; P<$ .05 ) or excluded (young: mean 2.2, SD 0.4; old: mean $2.9 \mathrm{sec}$, SD 0.6; $P<.005$ ). Despite the age group difference in task completion time, time to rise did not correlate significantly with the presence or absence of any of the three descriptor items that differed between the young and old (such as trunk lateral flexion/rotation).
Table 1. Supine-to-Sit (SS) Task Descriptors Present in Young Versus Old (\% of group)

\begin{tabular}{|c|c|c|c|}
\hline Descriptor & Early & Middle & Late \\
\hline \multicolumn{4}{|c|}{$\begin{array}{l}\text { Trunk Motion (by pivot phase) } \\
\text { Trunk flexion in sagittal } \\
\text { plane }\end{array}$} \\
\hline Young & 90 & 45 & 5 \\
\hline $\begin{array}{l}\text { Old } \\
\text { Trunk lateral flexion } \\
\text { rotation }\end{array}$ & 79 & 29 & 0 \\
\hline $\begin{array}{l}\text { Young } \\
\text { Old }\end{array}$ & $\begin{array}{l}10 \\
28\end{array}$ & $\begin{array}{l}25 \\
50\end{array}$ & $\begin{array}{l}30 \\
71^{\star}\end{array}$ \\
\hline $\begin{array}{r}\text { Pivot variants (by trun } \\
\text { elevation phas } \\
\text { Weight bear on left } \\
\text { hip/gluteal aree }\end{array}$ & & & \\
\hline Young & 5 & 25 & 100 \\
\hline $\begin{array}{l}\text { Old } \\
\text { Broad base (hip/elb } \\
\text { pivot }\end{array}$ & 29 & $72^{\star \star \star *}$ & 86 \\
\hline $\begin{array}{l}\text { Young } \\
\text { Old }\end{array}$ & $\begin{array}{r}5 \\
24\end{array}$ & $\begin{array}{l}15 \\
64^{\star \star}\end{array}$ & $\begin{array}{r}5 \\
21\end{array}$ \\
\hline
\end{tabular}

Descriptor can be rated as present or not present, during the early, middle, and/or late phases of either trunk elevation or pivor completion. Young $(n=2)$ and Old $(n=3)$ subjects performing rolls onto their ipsilateral side were excluded from the analysis.

Percent of Old $(n=14)$ different than Young $(n=20):{ }^{*} P<.05 ;{ }^{*} P<.01$; $* * * P<.005$.

\section{Supine-to-Sit Initiation}

The percentage of use of a body segment to initiate the SS task was noted (see Table 2), and these data include subjects who rolled onto their side to rise. The young adults initiated the SS task with only neck flexion more often than the old, whereas the old initiated the SS task with only upper extremity motion more often than the young. The old tended to demonstrate more variability in initiating the SS task with neck flexion only, upper extremity motion only, or lower extremity motion only.

Table 2. Body Segment Motion Initiators for SS Task in Young and Old

$\begin{array}{cc}\begin{array}{c}\% \text { of Young } \\ (n=22)\end{array} & \begin{array}{c}\% \text { of Old } \\ (n=17)\end{array}\end{array}$

$\begin{array}{lrc}\text { Neck flexion only } & 68 & 24^{*} \\ \text { Upper extremity motion only } & 9 & 41^{\circ} \\ \text { Lower extremity motion only } & 9 & 18 \\ \begin{array}{l}\text { Neck flexion and upper } \\ \quad \text { extremity }\end{array} & 0 & 6 \\ \begin{array}{l}\text { Neck flexion and lower } \\ \quad \text { extremity }\end{array} & 9 & 0 \\ \begin{array}{l}\text { Upper and lower extremity } \\ \text { Neck flexion, upper and lower }\end{array} & 5 & 6 \\ \text { extremity } & 0 & 6\end{array}$

Percent of Old adults different than Young adults: ${ }^{*} P<.05$. Subjects who performed rolls are included in this sample (see text). 


\section{"Sit-up" Performance and Impact on Supine-to-Sit Data}

All young and old subjects were able to complete the SUH task. All of the young but only 6 of 17 of the older adults (35\%) were able to complete the SUNH task (young-old difference, $P<.001$ ). When excluding the roll subjects, 6 of $14(43 \%)$ of the old were able to complete the SUNH task (young-old difference, $P<.001$ ).

Excluding the roll subjects, the subgroup of older women who were unable to complete the SUNH task, the old unable $(n=8)$, when compared with the older women who could complete this task, the old able $(n=6)$, had a tendency to differ in their SS task descriptors (Table 3). The old unable, compared with the old able, more often tended to use lateral trunk flexion, weight bearing on the left hip, and a broader support base with their elbows, although these differences were not statistically significant. The old unable initiated the SS task significantly more often with the upper extremity than with neck flexion. SS task completion time did not differ significantly between the old able (mean $2.8 \mathrm{sec}$ ) and the old unable (mean $3.0 \mathrm{sec}$ ). When examining Table 1 , Table 2 , and Table 3 more carefully, the descriptors that differentiated the young and the old groups also tended to differentiate the old able and old unable. This suggests that the old unable subset may have accounted for much of the young-old descriptor differences seen previously. The old able did not differ from the old unable in the presence or absence of subtle history and examination abnormalities (approximately half of each group).

\section{Reliability}

Using the individual ratings for the SS task that differed between young and old adults (see Tables 1 and 2), Cohen's Kappa scores for interrater, intrarater, and intertrial agreement were analyzed. Interrater agreement was excellent (ranging from 0.73 to 0.94 ), except for the late pivot phase of trunk lateral flexion/rotation (0.28). Intrarater and intertrial agreement were also excellent (ranging from 0.79 to 1.0 ).

\section{DISCUSSION}

Compared with young women, older women with no apparent bed mobility difficulty differ in the trunk and pivot motions used in rising from supine to sitting, despite only minimal differences in rise time. The older women apparently avoid trunk elevation in the sagittal plane by using trunk lateral flexion and rotation. Trunk elevation and pivoting is facilitated in the older adults by bearing weight on the hip/ gluteal area earlier and more often, and by using the elbow to broaden the base of support. Surprisingly few older adults choose to roll onto their sides and then rise, a strategy that has been previously assumed to be common among older adults. $^{5}$

Fewer than half of the older women could sit up in bed without the use of their hands. Older subjects who were unable to perform the SUNH task were generally the same older subjects who differed from the young in SS task items such as motion initiation, trunk flexion, and use of a broader support base. Thus, age-related decrements in trunk flexion ability may have accounted for the age-related differences seen in the SS task.

Movement patterns used to rise from a supine position are variable. ${ }^{9-13}$ Variability in initiation of body segment motion was present in the SS task, particularly in the older women. Studies involving aged populations have commonly reported large performance variations. ${ }^{21}$ A larger sample size may yield more significant age group differences, and some of these differences may be important clinically. We attempted to identify the most important SS descriptor items, given the subject sample size constraints and the effort required to acquire and analyze the data.

The SUNH task is similar to standard tests of abdominal muscle strength, the primary contributor to trunk flexion strength. ${ }^{22}$ Investigation of age-related decrements in trunk flexion strength over the range of motion similar to that required for rising from a supine to sitting position, to our knowledge, has not been performed. ${ }^{23-28}$ In order to evaluate the importance of trunk flexion in age-related changes of bed mobility, future studies of supine-to-sit tasks should consider direct measurements of trunk flexion strength and range of motion.

Nevertheless, trunk flexion strength measurements may relate only weakly to the ability to rise from supine-tositting. ${ }^{29}$ This weak relationship may be due to the use of muscle groups other than the abdominals, such as in the legs, ${ }^{30}$ to complete trunk flexion beyond 45 degrees of elevation $^{31}$ and because of the trunk coordination and balance required to rise from supine to sitting. ${ }^{32}$ Perhaps age-related declines in maintaining trunk balance ${ }^{33}$ thus contribute to age-related differences in supine-to-sit performance.

Could bed mobility differences between the young and old be related to subtle, underlying disease or inactivity? One study reports that differences in physical activity level influence how a middle-aged subject rises from a supine position

Table 3. Differences in SS Task Descriptors Between Old Able or Unable To Do No-Hand Sit-up

Descriptor $\quad \%$ of Able $(n=6) \quad \%$ of Unable $(n=8)$

Neck/Trunk Motion: Trunk lat flex/rotate

Late phase pivot

50

88

Pivot Variants: Weight bear on $L$ hip/gluteal area

Middle phase trunk elevation

50

Middle phase trunk elevation

Body Segment Motion Initiator

Neck flexion only

Upper extremity motion only
67

0
88

$0^{*}$

$75^{\star}$

Percent of Able different than Unable: ${ }^{*} P<.05$. 
on the floor to a standing position. ${ }^{34}$ Although generally healthy and active, a few of the older women had abnormal history and examination findings. Yet these findings did not affect their performance, such as in the SUNH task. These highly motivated women may, in fact, be unusually good performers, and repeat studies may find greater differences between young and older adult groups

\section{Clinical and Scientific Relevance}

As an initial study, the SS descriptor format, using body segment patterns instead of a more objective format such as joint angle measurement, seemed more appropriate in assessing complex 3-dimensional bed mobility. These motion patterns can be used in biomechanical analyses of the strengths and ranges of motion required to perform these tasks. For example, when rising from bed, how do trunk strength and range of motion requirements change when a patient flexes or rotates the trunk primarily laterally? What are the consequences, in terms of changes in center of pressure location, when trunk balance during a supine-to-sit pivot is supplemented by elbow contact?

Biomechanical analyses fit well with dynamic systems approaches to movement analysis. ${ }^{35}$ Variables affecting the performance of the bed mobility task, such as strength and joint range of motion, can be varied in a computer model to determine critical parameter values. Of particular importance is to test these parameters during periods of instability or transition, such as during trunk elevation and pivoting.

These analyses can also serve as a basis for developing age-appropriate goals and therapeutic exercises for bed mobility rehabilitation. The finding that some older adults cannot do a sit up without hand use means that their trunk flexion ability has declined. This decrement in trunk flexion may have an important impact in situations where compensatory adaptations are difficult, such as when extremity use is limited by fracture, weakness, or joint pain.

\section{ACKNOWLEDGMENTS}

The authors thank Albert B. Schultz, PhD, Janet Grenier, Becky Luebke, Kathy Mulligan, Teresa Norris, Michael Ward, and Linda Folkmier for their assistance in completing this project. The authors also thank the Claude Pepper Geriatrics Research and Training Center and Turner Geriatrics Clinic for their assistance and cooperation.

\section{REFERENCES}

1. Leon J, Lair T. Functional status of the noninstitutionalized elderly: Estimates of ADL and IADL difficulties, DHHS Publication No. (PHS)90-3462. National Medical Expenditure Survey Research Findings 4, Agency for Health Care Policy and Research. Rockville, MD: Public Health Service.

2. Dawson D, Hendershot G, Fulton J. National Center for Health Statistics. Aging in the eighties. Functional limitations of individuals age 65 and over. Advance data From Vital and Health Statistics, No.133. DHHS Pub. No. 87-1250. Hyattsville, MD: Public Health Service, June 10, 1987.

3. Katz S, Stroud MW. Functional assessment in geriatrics: A review of progress and directions. J Am Geriatr Soc 1989;37:267-271.

4. Schultz AB, Alexander NB, Ashton-Miller JA. Biomechanical analyses of rising from a chair. J Biomech 1992;25:1383-1391.

5. Carr JH, Shepherd RB. A Motor Learning Programme for Stroke, 2nd Ed. London: Heinemann, 1987.

6. Voss D, Ionta MK, Myers BJ. Proprioceptive Neuromuscular Facilitation: Patterns and Techniques, 3rd Ed. Philadelphia: Harper and Row, 1985.
7. Fiorentino MR. Reflex Testing Methods of Evaluating CNS Development, 2nd Ed. Springfield: C.C. Thomas, 1973.

8. Davies PM. Steps to Follow: A Guide to the Treatment of Adult Hemiplegia. New York: Springer-Verlag, 1985.

9. VanSant AF. Rising from a supine to erect stance: Description of adult movement and a developmental hypothesis. Phys Ther 1988;68:185-192.

10. VanSant AF. Age differences in movement patterns used by children to rise from a supine to erect stance. Phys Ther 1988;68:1330-1339.

11. VanSant AF. Life-span development in functional tasks. Phys Ther 1990;70:788-798.

12. Richter RR, VanSant AF, Newton RA. Description of adult rolling movements and hypothesis of developmental sequences. Phys Ther 1989;69:6371.

13. McCoy JO, VanSant AF. Movement patterns of adolescents rising from a bed. Phys Ther 1993;73:182-193.

14. Jebsen RH, Trieschmann RB, Mikulic MA et al. Measurement of time in a standardized test of patient mobility. Arch Phys Med Rehabil 1970;51:170175.

15. Alexander NB, Fry-Welch D, Ward ME, Folkmier LC. Quantitative assessment of bed rise difficulty in young and elderly women. J Am Geriatr Soc 1992;40;685-691.

16. Kottke FJ, Stillwell GK, Lehmann JF. Krusen's Handbook of Physical Medicine and Rehabilitation, 3rd Ed. Philadelphia: WB Saunders, 1982.

17. DeJong RN. The Neurologic Examination, 4th Ed. Hagerstown: Harper and Row, 1979.

18. Polley HF, Hunder GG. Rheumatologic Interviewing and Physical Examination of the Joints, 2nd Ed. Philadelphia: W.B. Saunders, 1978.

19. Feinstein AR. Clinimetrics. New Haven: Yale University Press, 1987.

20. Wilkinson L. Systat: The system for statistics. Evanston: SYSTAT, Inc., 1987.

21. Zimmer AW, Calkins $\mathrm{E}$, Hadley $\mathrm{E}$ et al. Conducting clinical research in geriatric populations. Ann Intern Med 1985;103:276-283.

22. Daniels L, Worthingham C. Muscle Testing: Techniques of Manual Examination, 4th Ed. Philadelphia: W.B. Saunders, 1980.

23. McNeil T, Warwick D, Andersson G, Schultz A. Trunk flexion in attempted flexion, extension, and lateral bending in healthy subjects and patients with low-back disorders. Spine 1980;5:529-538.

24. Viitasalo JT, Era P, Leskinen AL et al. Muscular strength profiles and anthropometry in random samples of men aged 31-35, 51-55, and 71-75 years. Ergonomics 1985;28:1563-1574.

25. Sandler RB, Burdett R, Zaleskiewicz $M$ et al. Muscle strength as an indicator of the habitual level of physical activity. Med Sci Sports Exerc 1991;23:1375-1381.

26. Sparup KH. Late progress in lumbar disc herniation. XII. Muscle Testing. Acta Rheuma Scand 1960;3 (Suppl):130-137.

27. Hasue M, Fujiwara M, Kikuchi S. A new method of quantitative measurement of abdominal and back muscle strength. Spine 1980;5:143-148.

28. Helewa A, Goldsmith $\mathrm{C}$, Smythe $\mathrm{H}$ et al. An evaluation of four different measures of abdominal muscle strength: Patient, order and instrument variation. J Rheumatol 1990;17:965-969.

29. Gersten JW, Ager C, Anderson K et al. Relation of muscle strength and range of motion to activities of daily living. Arch Phys Med Rehabil 1970;51:137-142.

30. Ricci B, Marchetti M, Figura F. Biomechanics of sit-up exercises. Med Sci Sports Exercise 1981;13:54-59.

31. Flint MM. Abdominal muscle involvement during the performance of various forms of sit-up exercise. Am J Phys Med 1965;44:224-234.

32. Miller MI, Medeiros JM. Recruitment of internal oblique and transversus abdominis muscles during the eccentric phase of the curl-up exercise. Phys Ther 1987;8:1213-1217.

33. Alexander NB, Shepard N, Gu MJ, Schultz AB. Postural control in young and elderly adults when stance is perturbed: Kinematics. J Gerontol 1992;47:M79-87.

34. Green LN, Williams K. Differences in developmenal movement patterns used by active versus sedentary middle-aged adults coming from a supine position to erect stance. Phys Ther 1992;72:560-568.

35. Giuliani CA. Theories of motor control: New concepts for physical therapy. In: Contemporary Management of Motor Control Problems. Proceedings of the IIStep Conference. Foundation for Physical Therapy, Section on Neurology and Pediatrics, APTA, 1991. 